\title{
Sorting Terms of "aaS" of Everything as a Service
}

\author{
Yucong Duan ${ }^{1}$, Xiaobing Sun ${ }^{2}$, Antonella Longo ${ }^{3}$, Zhaoxin $\operatorname{Lin}^{4}$, Shixiang Wan ${ }^{1}$ \\ ${ }^{1}$ Information Science and Technology College, Hainan University, China \\ E-mail: duanyucong@hotmail.com,shixiangwan@gmail.com \\ ${ }^{2}$ School of Information Engineering, Yangzhou University, China, \\ E-mail: sundomore@163.com \\ ${ }^{3}$ Department of Engineering for Innovation, Universit del Salento, Italy, \\ E-mail: antonella.longo@unisalento.it \\ ${ }^{4}$ Department of Information System and Operation Management, University of Florida, USA, \\ E-mail: zxlin@ufl.edu
}

\begin{abstract}
Numerous service models have been proposed in the form of "as a Service" or "aaS" in the past. This is especially eminent in the era of the Cloud under the term of anything as a service or everything as a service(XaaS). A unified view of XaaS can aid efficient classification of services for service registration, discovery, and composition. In view of that there lacks such a unified view which is demanded by systematic application of XaaS in the Web Service Ecosystem, towards proposing a taxonomy of "aaS", we present in this work a collection of "aaS" based on a throughout literature survey. We also proposed a strategy to identify the terms from the servicelization perspective.
\end{abstract}

Keywords: Everything as a Service; Anything as a Service; Cloud Computing; SOA

\section{Introduction}

Various emerging trends especially Cloud Computing [6] and Big Data analysis can be increasingly identified by different "as a Service (aaS)" in providing everything as a service(XaaS) $[36 ; 75]$. In the past years related terms are used in a discretionary way creating some entropy $[36 ; 36 ; 91]$. This should be avoided with unified classification. Based on the hypothesis that the classification under the name of "aaS" partially reflects the trends of natural evolution of services sharing common characteristics, we present in this work our effort of literature survey towards describing a technical classifi- cation of various "aaS". The survey covers Google, DBLP, IEEE Xplore Digital Library and ACM Digital Library mainly including keywords like "as a service" and "as-a-service" varied from 1984 to 2014. The survey covers literature with content including explicitly focused "aaS" marked with "E", mentioned "aaS" marked with "M" and implicitly derived "aaS" marked with "I" from traditional areas to IT applications. We expect this work can initiate a profound discussion on the future trend of business service opportunities, IoT service development, trend of service migration to Cloud, and Big Data service analysis. 


\section{Various "aaS"}

The abbreviation of a specified service is referred from the surveyed paper. We filtered those only mentioned a term of "aaS" without elementary discussion. Figure 1 shows the distribution of articles. Most of the surveyed papers are from proceedings or journals. A few of them are technical reports and books. To sum up, we list in the following paragraphs all the categories of "aaS" which include 144 cases of "aaS".

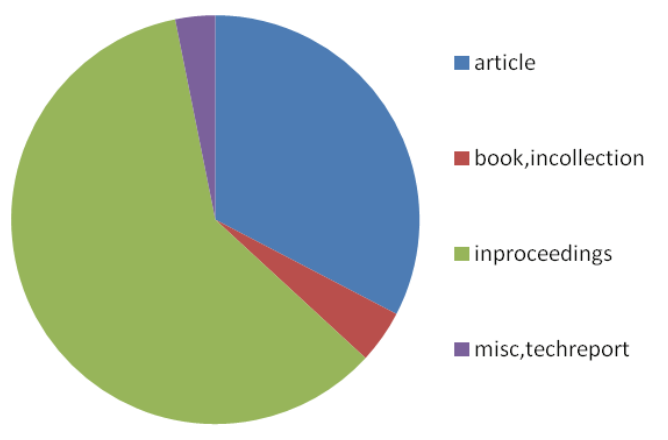

Fig. 1. Distribution of literatures

\subsection{Traditional services}

A lot of ordinary activities have been identified as either an intangible commodity with economical meaning or a means of serving others through resources, skill, ingenuity, and experience. Their identification forms a process of cognitive progress which lays the cognitive foundation for modern XaaS in Cloud. Chronologically we collect 9 traditional "aaS" as follows. "Information as a Service (E)" (1984), Ives and Learmonth [49] proposed that information technology can help to reduce cost and win the market competition. "Teaching Mathematics as a Service (E)"(1988), Howson et al. [47] proposed teaching mathematics as a service subject. "Ownership as a Service (M)" (1997), Beaumont et al. [9] classified ownership of dwellings as an intangible economic goods. "Consulting as a Service (D)" and "Education as a Service (D)" and "Knowledge Management as a Service (E)" (1999), Kailer and Scheff [53] proposed consulting, education and knowledge management as a Service. "Health as a Service (D)" and "Telemedicine as a Service (D)"
(2001), Edworthy [34] took Health service the superclass of tele-medicine service. Similarly Fano and Gershman [37] proposed "Medical Care as a Service (M)". Print as a Service (D) and "Quote as a Service (D)" (2003), Perrey and Lycett [72] mentioned that provision of printing and quote as a service. Since they are of value to the customer. "Internet as a Service (E)" (2006), van Deursen and Pieterson [86] proposed Internet as a Service Channel. "Washing Machine as a Service (D)" (2007), Bottaro et al. [15] proposed that washing machine may be considered as a service with a stateful behaviour. "Music as a Service (E)" 2010, Doerr et al. [30] proposed it as a new concept of digital music distribution which enables music streaming over the Internet without transferring ownership for the content.

\subsection{Network services}

From the literature survey, we collected 10 network related "aaS" as follows. "Data Mining Models as a Service (E)" (2000), Sarawagi and Nagaralu [77] proposed it to cover Document Classification service, Collaborative Filtering Service, and Risk Prediction service. "Authentication as a Service (E)" (2005), Laitinen et al. [57] leveraged authentication from a communication infrastructure to a service provision in Cellular Network. Similarly Lakshminarayanan et al. [58] proposed "Routing as a Service (E)" (2006) to leverage specialized route computation as a service. "Accountability as a Service" (2007), Bender et al. [11] isolated and took accountability as a network traffic service. In 2014, Bitterman et al. [13] proposed Integrated Authentication, Authorization and Accounting (AAA) services provision through a common Active Directory server. "Network Protocol as a Service (M)" (2011), Feng et al. [38] proposed Networking as a Service by combining the service provision model of cloud computing with the openness of the network protocol. "Routing as a Service (RaaS) (E)" (2011), Chen et al. [21] proposed it as a framework for tenantDirected route control in data center. "IP Networks as a Service (E)" and Virtual Routers as a Service (E)] (2011), Nascimento et al. [64] proposed that virtual routers and IP networks as a service. "Hybrid 
Network as a Service (HaaS) (E)" (2011), Mizusawa and Kitsunezaki [63] proposed a Hybrid Network service combining a dark fibre network (nonIP) with IP network.

\subsection{Services in programming/modelling}

We identified 8 programming and modeling related "aaS" as follows. "Virtual CPU as a Service (M)" (2003), Figueiredo et al. [39] proposed that CPU farm may provide the capability of instantiating fullblown virtual back-ends as a service under Operating System Terminology. "Coordination as a Service (E)" (2003), Viroli and Omicini [88] proposed it as providing a convention of programming in geography software. "Communication as a Service (M)" and "Function as a Service (M)" and "Program Call as a Service (M)" (2004), Dunkels et al. [32] designed a program in which communication is implemented as a service in order to enable run-time replacement and program call was implemented through the service interface stub. A particular function was implemented as a service. "Data Access as a Service (D)" (2007), Dan et al. [28] proposed that the data access service can be easily developed by a DBA using high-level tools. "Component as a Service (D)" and "Functionality as a Service (D)" (2009), Jansen et al. [50] pointed out that components was eventually to be published as a service which represents software functionality to be run independently. "Software as a Service (SAAS) (E)" (2000), Bennett et al. [12] proposed this term which is different from the later more popular term of "SaaS". "Database Management as a Service (E)" (2002), Hacigumus et al. [44] proposed Database as a Service a new paradigm for data management in which a third party service provider hosts the database and provides its customers seamless mechanisms to create, store, manage, and access their databases. "Business Process as a Service (D)" or "Transactions as a Service (D)" (2003), Papazoglou [67] proposed to extend the software as a service concept to include the delivery of complex business processes and transactions as a service

\subsection{General $\mathrm{SOA}$ services}

We collected 11 SOA related "aaS" as follows. "Knowledge as a Service (E)" (2005), Xu and Zhang [95] introduced knowledge as a service in which a knowledge service provider answers queries presented by some knowledge consumers. "Application as a Service (M)" and "Database as a Service (M)"'(2005), Xu and Zhang [95] mentioned that application and database was provided as a service was under SOA. "Information as a Service (IaaS) (E)" (2007), Dan et al. [28] applied SOA principles to enable the utilization of Information as a Service (IaaS). "Operating System as a Service (E)" (2007), Milanovic and Malek [62] proposed a framework for construction of "service-oriented operating system" as a set of collaborating services. "Function as a Service (D)" (2007), Papazoglou et al. [68]proposed to enable reusable application functions as services. "Data as a Service (M)" (2008), Dwivedi and Kulkarni [33] mentioned a scenario of Data as a Service. "Data Analytics as a Service (M)" (2008), Dwivedi and Kulkarni [33] presented the key complexities and architecture involved a SOA realization scenario of Data Analytics as a Service. "Functionality as a Service (D)" (2009), Patel et al. [69] talked the paradigm in which every functionality is provided as a service that may not necessarily come from the same provider. "Process as a Service (E)"(2010), Wang et al. [89] proposed it based on that a provider may expect a process to be available serviceable for more process clients. "Models as a Service (MaaS) (E)" (2010), Brunelire et al. [16] pointed out that the ENVISION project has a meaning of Models as a Service. The Models as a Service is a way to exchange environmental models. "Cashier as a Service (CaaS) (E)" (2011), Wang et al. [90] referred merchant websites that accept payments through thirdparty cashiers as Cashier-as-a-Service.

\subsection{Web Services}

Chronologically we collected 9 Web Services related "aaS" as follows. "Computational Resource as a Service (E)" (2002), Furmento et al. [40] implemented Computational Resource as a Service as an OGSA compliant Web Service. "Web Service 
Composition as a Service (D)" (2003), Sirin et al. [80] proposed Web Service Composition online as a service. "Experiments as a Service (E)" (2005), Ott et al. [66] implemented Experiments as Web services, or use Web Service as an optional interface. "Industrial Machines as a Service (IMaaS)(E)" (2006), Gilart-Iglesias et al. [41] proposed Industrial Machines as a Service based on Embedded Devices and Web Services. "Identity as a Service (E)" (2007), Emig et al. [35] discussed how to construct an SOA-aware Identity management (IdM) architecture enabling "Identity as a Service" "Flexibility as a Service (FAAS) (E)" (2009), van der Aalst et al. [85] addressed the need for flexibility and provision Flexibility as a Service(FAAS). "E-assessment as a Service (E)" (2011), Amelung et al. [5] implemented it as an e-learning tool to benefit from automatic assessment. "Modeling as a Service (D)" and "Training as a Service (D)" (2014), Bitterman et al. [13] pointed out that Polymer Portal, a first-generation simulation as a service, was able to integrate access to multiple modeling and training services. "Laboratories as a Service (LaaS) (E)" (2014), Caminero et al. [18] proposed the creation of Laboratories as a Service (LaaS)which allows the use of remote laboratories to consumed from third parties.

\subsection{The Cloud era}

We list the Cloud related "aaS" in the following subsections.

\subsubsection{Infrastructure as a Service}

We collected 8 infrastructure related "aaS" as follows. "Storage as a Service (E)" (2009), Grossman et al. [43] proposed it for a storage cloud. "Hardware as a Service (M)" and "IT Infrastructure Management as a Service (M)" (2009), Kaufman [55] proposed provision hardware as a service and IT Infrastructure as a service. "Communication as a Service (M)" and "computing as a Service (M)" (2010), Tsai et al. [84] showed that communication and computing was served in infrastructure layer. "Virtual Machine as a Service (D)" (2010), Wu et al. [94] proposed providing access to VMs themselves as a service. "Resource as a Service (RaaS) (E)"
(2012), Agmon Ben-Yehuda et al. [1] termed this as a nascent economic model of cloud computing. "Secure Communication as a Service (D)" (2011), Peraković et al. [70] proposed secure communication with services such as police, emergency medical service, fire department. "Media Network as a Service (MNaaS) (E)" (2014), Cicic and Elmokashfi [26] presented it to combine a high-quality network infrastructure with a feature-rich video-conferencing infrastructure. "Security as a Service (E)" (2014), Varadharajan and Tupakula [87] contributed a security architecture that provides flexible security services.

\subsubsection{Platform as a Service}

We collected 10 platform related "aaS" as follows. "Hardware as a Service (HaaS) (E)", Platform as a Service (PaaS) E) (2008), Aymerich et al. [7] identified Hardware as a Service (HaaS) and Platform as a Service (PaaS) as Cloud Service. Database Management as a Service (E) (2009), Agrawal et al. [2] proposed it for data management. "Development as a Service (M)", Modeling as a Service (M), and Testing as a Service (M) (2010), Tsai et al. [84] identified modeling, development, and testing services as PaaS services. "Application as a Service (M)", Business Process as a Service (M), and UML Modeling as a Service (2009), Kaufman [55] categorised application services, UML Modeling tools services and social network services into an application Cloud service. "Disaster Recovery as a Service (E)" (2010), Wood et al. [93] proposed that offering Disaster Recovery (DR) as a service to their pay-asyou-go pricing model. "IDE And Hosting as a Service (E)" (2011), Aho et al. [3] described providing the IDE and hosting as a service. "Database as a Service (E)" (2011), Howe et al. [46] proposed it. "Continuous Analytics as a Service (CaaaS) (E)" (2011), Chen et al. [23] proposed providing Continuous Analytics as a Service (CaaaS) and implemented CaaaS as Software as a Service as well as Platform as a Service. "OLTP Database as a Service (M)" and Parallel Database as a Service (M) (2013), Wong et al. [92] proposed it to support a large number of concurrent query executions after consolidation. "Content Delivery as a Service (CoDaaS) (E)" (2014), Jin 
et al. [51] proposed it to distribute user generated content (UGC) in an efficient and economical fashion.

\subsubsection{Software as a Service}

We collected 20 "SaaS" cases as follows. "Software as a Service (SaaS) (E)" (2008), Aymerich et al. [7] proposed Software as a Service (SaaS), as a kind of Cloud Service. "Custom Relationship Management as a Service (M)" (2009), Kaufman [55] identified Custom Relationship Management as a SaaS. "Middleware as a Service (M)" (2009), Kaufman [55] identified "Middleware as a Service" as a SaaS. "Videoconference as a Service (VaaS) (E)" (2009), Rodríguez et al. [76] proposed providing videoconference as a web service in Cloud environment. "Commerce as a Service (CaaS) (E)" (2009), Cai et al. [17] proposed it centering around customer business requirements. "Social Network as a Service (SNaaS) (E)" (2009), Maamar and Badr [61] proposed a multi-tenant architecture to develop Social Networks-as-a-Service (SNaaS) and allow efficient use of server resources. "Data as a Service (E)" (2009), Truong and Dustdar [82] pointed out that various research effort have concentrated on the development of the concept of provision data/information as a service. "Automated Software Testing as a Service (TaaS) (E)" (2010), Candea et al. [19] made the case for TaaS-automated software testing as a Cloud-based service. "Design as a Service (M)" Email as a Service (M), ERP as a Service (M), Office as a Service (M), User Interface as a Service (M) (2010), Tsai et al. [84] identified design, e-mail, office and user interface services as Software as a Service. "Testing as a Service (E)" (2010), Yu et al. [97] proposed it as a new model to provide testing capabilities to end users. "Application Based Paas as a Service (aPaaS) (E)" (2011), Beimborn et al. [10] called it that a software firm offers a core application aPaaS (application based PaaS). "Risk Assessment as a Service (E)" (2010), Kaliski Jr and Pauley [54] recommended addressing potential risks and exposures by introducing risk assessment as a service. "Authentication as a Service (AaaS) (E)" (2011), Senk and Dotzler [78] proposed it if security-related services is obtained from an external provider. "Biometric Authentication as a Service (BioAaaS) (E)" (2011), Senk and Dotzler [78] proposed Biometric Authentication as a Service (BioAaaS) as an innovative approach for strong authentication in web environments based on the Software as a Service model. "Exploit as a Service (E)" (2012), Grier et al. [42] investigated the emergence of it as an application of software-asa-service paradigm for drive-by browser compromise. "Component as a Service (E)" (2013), La et al. [56] proposed it to provision a reusable functionality which is subscribed by applications and fulfils the functionality needed by the applications. "Simulation as a Service (SMaaS) (E)" (2014), Bitterman et al. [13] extended SaaS to include high performance computing-hosted applications, and creating Simulation as a Service (SMaaS). "Business Analytics as a Service (BAaaS) (E)" and "Business Intelligence as a Service (BIaaS) (E)" (2014), Chang [20] described it as Cloud based services designed to present financial data and improve the accuracy and quality of both pricing and risk analysis in financial markets in a collaborative and easy-tounderstand style. "Heston Volatility And Pricing as a Service (HVPaaS) (E)" (2014), Chang [20] also demonstrated Heston Volatility and Pricing as a Service (HVPaaS) to process stochastic equations and present the calculated implied volatility and pricing. "Hospital Information Software as a Service (HI-SaaS) (E)" (2014), Yao et al. [96] established a Cloud-based Hospital Information Service Center to provide hospital information software as a service.

\subsubsection{Other classification}

We classified other 28 the Cloud related "aaS" as follows. We put some "aaS" here since they could be implemented covering several layers of the above classification. "Everything as a Service (E)(2008), Robison et al. [75] used "Everything as a Service" to call the future that everything will be delivered as a service mostly via Internet. "Privacy as a Service (PasS) (E)" (2009), Itani et al. [48] presented it as a set of security protocols for ensuring the privacy and legal compliance of customer data in cloud computing architectures. "Computing Resources as a Service (M)" (2009), Patel et al. [69] proposed that 
readily available computing resources are exposed as a service. "Infrastructure as a Service (IaaS) (M)" Business as a Service (BaaS) (M), Database as a Service (DaaS) (M), Desktop as a Service (DaaS) (M), Development as a Service (DaaS) (M), Framework as a Service (FaaS) (M), Organization as a Service (OaaS) (M) (2009), Rimal et al. [74] used infrastructures, business, database, desktop, development, framework, organization services as examples of "X" as a Service. "Search as a Service (E)" (2009), Singh et al. [79] proposed to provide search indices to be hosted at the SSP site which effectively provides search-as-a-service. "Information Acquisition as a Service (E)" (2010), Craciunas et al. [27] proposed it for mobile sensor networks. "Component as a Service (M)" (2010), Dawoud et al. [29] proposed that Cloud Computing intersects with SOA in Components as a Service. "Education and Learning as a Service (ELaaS) (E)" (2011), Alabbadi [4] discussed the use of cloud computing in the educational and learning area. "IT as a Service (ITaaS) (M)" (2011), Alabbadi [4] proposed it for application and IT users since Cloud Computing means provision IT as a Service (ITaaS). "IT Management as a Service (D)" (2011), Banerjee et al. [8] proposed that the IT management services is a delivering technologies. "Capabilities as a Service (D)" (2011), Subashini and Kavitha [81] referred that Gartner defines cloud computing as s style of computing where massively scalable IT-enabled capabilities are delivered "as a service". "Disaster Tolerance as a Service (E)" (2012), Rajagopalan et al. [73] proposed SecondSite, a high-availability and disaster tolerance service for virtual machines running in cloud environments. "Threat as a Service (E)" (2012), Tsai et al. [83] proposed it as an security service under Cloud environment. Forensics as a Service (E) Policing as a Service (E) and Privacy as a Service (2013), Zargari and Smith [98] classified Privacy As A Service and Forensics As A Service as sub services of "Policing as a Service". These services mainly concerns security and confidentiality of organizations that use cloud computing as a service infrastructure. "Telepresence as a Service (TPaaS) (E)" (2014), Cicic and Elmokashfi [26] proposed it as well as "TPaaS" (Telepresence as a Service). "E- commerce as a Service (D)" (2014), Bitterman et al. [13] implemented e-commerce services as front end interfaces for "Simulation as a Service". "Consistency as a Service (CaaS) (E)" (2014), Liu et al. [59] presented consistency as a service (CaaS) model, which consists of a large data cloud and multiple small audit clouds. "E-health as a Service (eHaaS) (E)" (2014), Black et al. [14] developed it as a datadriven extension to an integrated health record bank construct. "Analysis as a Service (E)" (2014), Lomotey and Deters [60] proposed that IBM Research has identified Analysis as a Service (AaaS) as an area that can offer business value. "Traffic Analysis as a Service (E)" (2014), Chu et al. [25] described a traffic analysis platform which enabled different government authorities or geographic locations to utilize its functionalities more efficiently and effectively.

Cloud Service Meet Big Data and Internet of Things Web of Things: "Things as a Service (E)] (2011), Christophe et al. [24] proposed the Web of Things, where things served as a Service and Interaction Patterns. "Environment as a Service" (2011), Christophe et al. [24] participated in Bell Labs "Environment as a Service" research theme within the Application Domain. "Big Data Platform as a Service (E)" (2012), Horey et al. [45] proposed it. "MPP Database as a Service (MPPDBaaS) (E)" (2013), Wong et al. [92] proposed that the offering of MPPDB-as-a-Service (MPPDBaaS) will become attractive for companies having analytical tasks on hundreds gigabytes to some ten terabytes of data. "Analysis as a Service (AaaS) (E)" (2014), Jingliang et al. [52] proposed that the case Big Data uses Cloud Computing platform was called AaaS (Analysis as a Service). Jingliang et al. [52] also proposed "Value as a Service (VaaS) (E)" (2014) for the case Cloud computing use Big Data and mentioned the challenging problem is how to discover the valuable service from Big Data. Data as a Service (DaaS) (E), Information as a Service (InaaS) (E), Knowledge as a Service (KaaS) (E) and Wisdom as a Service (WaaS) (E) (2014), Chen et al. [22] proposed a wisdom as a Service (WaaS) architecture of IT applications based on the DIKW hierarchy which includes Data as a service (DaaS), Information as a 
Service (InaaS), Knowledge as a Service (KaaS) and Widsom as a Service (WaaS). "Sensing as a Service (E)" (2014), Perera et al. [71] gave the concept of sensing as a service and how it fits with the Internet of Things.

Figure 2 shows the appearance of various "aaS" from traditional services to Cloud services. Although "aaS" is proposed dating back to the 1980s, we can observe that there is huge increase of the amount of proposed "aaS" after the emerging of the Cloud concept. The increase of the "aaS" at the Cloud era symbolizes the migration of the traditional services to the Cloud.

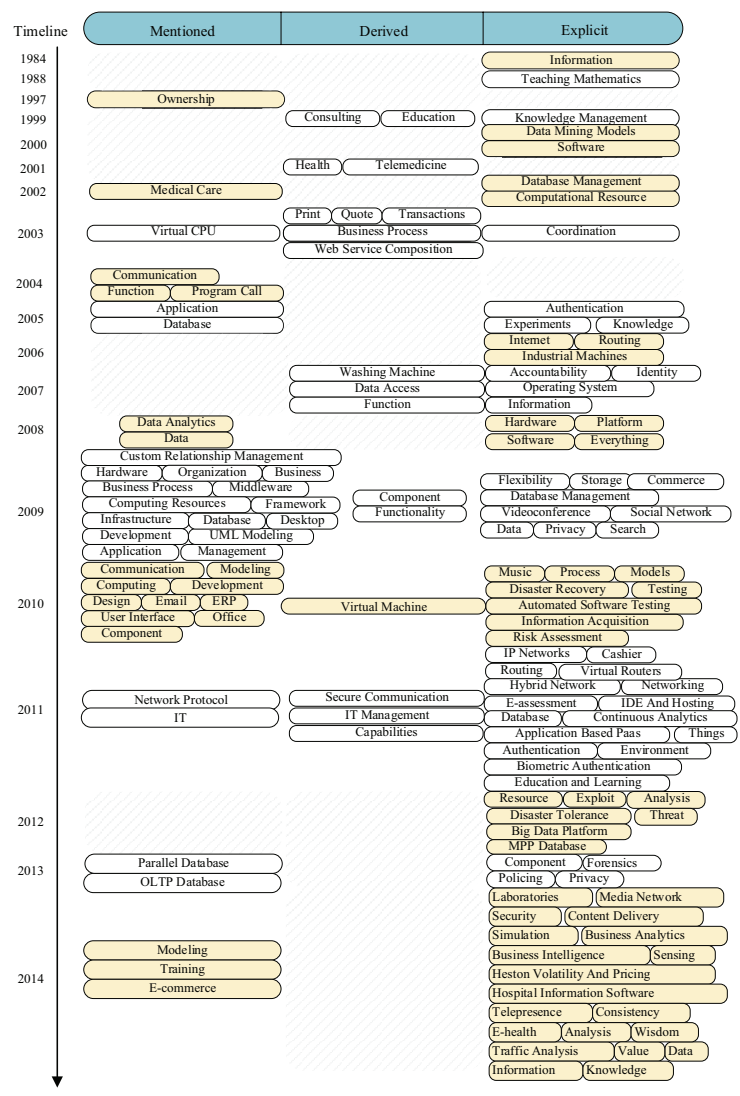

Fig. 2. The appearance of various "aaS" terms

\section{Conceptualization Based Sortting}

We tend to use these terms to represent the history of when various "aaS" are proposed and for what reasons they are proposed. The reasons might be a service migration from traditional business to the cloud as a provision revolution, or just a servicelizaition activity which identifies that a resource or activity or their compositions can be exchanged as a target/object of a transaction, etc. The distinguishment can be used to support analysis on service migration trends in terms of the amount of emerging "aaS", etc. After checking the background of various proposed "aaS", we found that the independent semantics of core words, which are used to name an "aaS", usually cannot fully identify whether it represents a service migration from traditional business to the Cloud, or it is only a servicelization. For example, when "Software as a Service" was proposed in 2000, it was a result of servicelization which identifies that software usage can be traded as a service. While "Software as a Service" was proposed in 2008, this term was intended to symbolize the utility of software in the Cloud. When we want to shape the trend of service migration to the Cloud in terms of "aaS", we clearly do not want to count the "aaS", which is a result of servicelization, as a case of migration to the Cloud. Therefore the meaning which we want to distinguish lies in the servicelization process when people name an "aaS". According to Figure 3, empirically we shape the process with the following stages:

- Conceptualization: an object or resource is recognized as an self-independent concept. An independent identity is achieved through the conceptualization process which cognitively labelled the intended content as a distinguishable identity, usually a concept which integrates the notation of the concept and the content represented by the concept. An example of the conceptualization is the identification of the "ownership" as a service.

- Externalization: externalization is a process of further separating a conceptualized target from a self serving state to a state served by a outsider whether it is a human being or a machine. For example, self education is not considered as a service for oneself but receiving education from others or through machines is a form of service consumption. Similarly printing by oneself is not a service but printing provided by others is a service.

- IT/Cloud service: IT services differ from traditional services in many aspects and the Cloud ser- 
vice is provisioned through the Cloud enabled service delivery models [36]. In [74], XaaS is initially proposed for the Cloud where $\mathrm{X}$ is software, hardware,platform, infrastructure, data, and business.

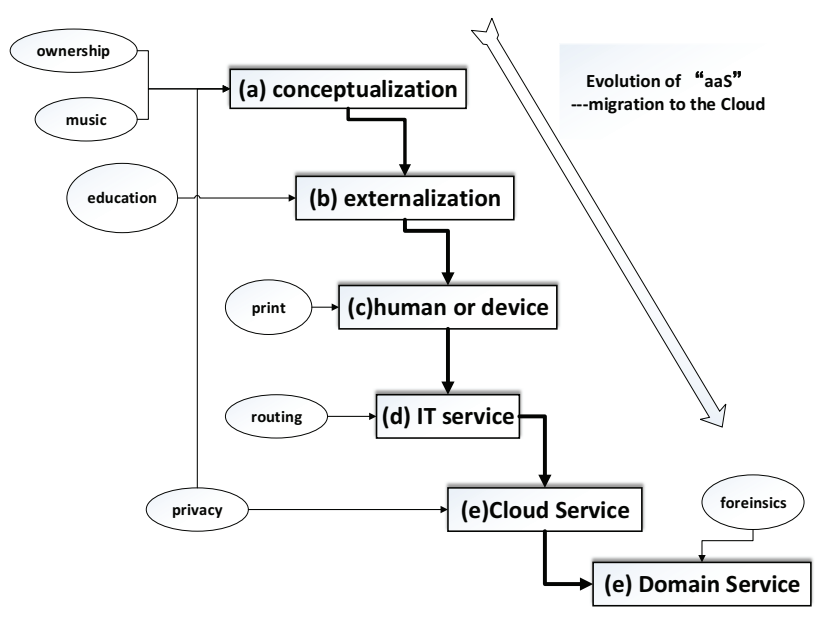

Fig. 3. The servicelization from concepts to the Cloud

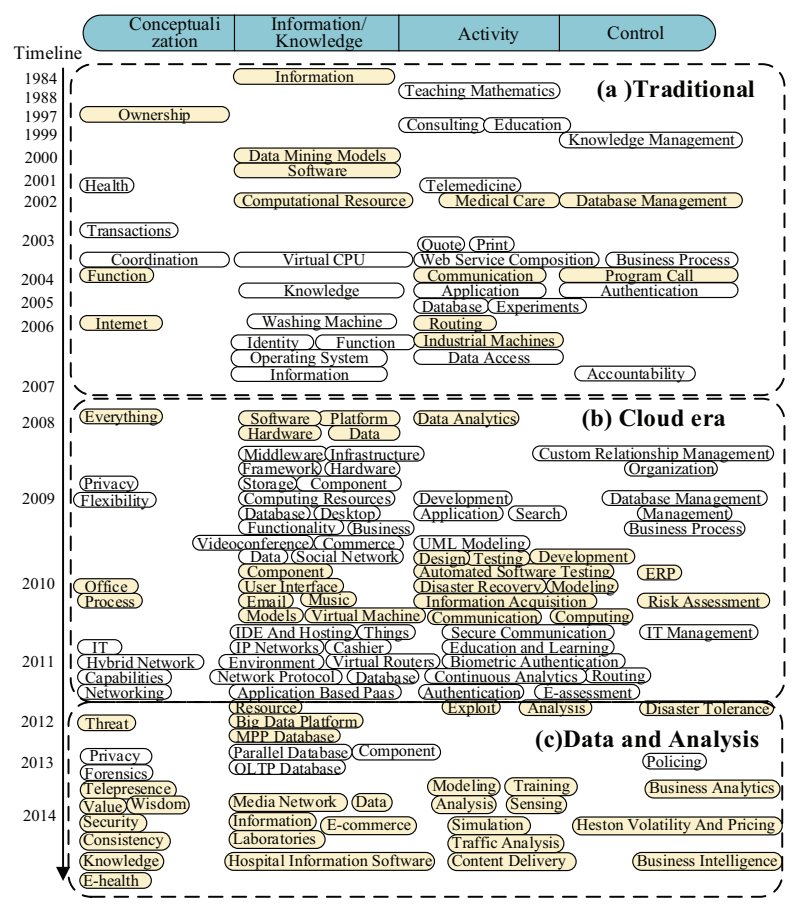

Fig. 4. Categorization on "aaS" terms and its application on migration trends
Figure 4 shows the result of a categorization following above sorting strategy. The column of "conceptualization" represents the "aaS" which is proposed to represent a source of services for the first time formally. The column of "Information/Knowledge" represents the externalized static resources which are used to label services, and the column of "Activity" represents the externalized actions and activities which are used to label services. The column of "Control" collects the "aaS" which is provided at a higher level as a service than basic activities. Based on the categorization, along the timeline we have found several interesting clues. In Figure 4, from 1984 to 2008, in all four categories we have found that various traditional services are conceptualized and externalized as commercial services. It symbolizes a servicelization process of traditional services. We marked this area with "(a) Traditional" in Figure 4. This servicelization stage lays the foundation for the expansion from traditional services to IT services and the Cloud. From 2008 to 2011, we have found a trend of virtualization on hardware, network, software, platform, middleware, framework, database, etc. We mark this virtualization trend with "(b) Cloud era". Following the virtualization of the Cloud area, we have identified the surge of the data analysis on various domains including forensics, business intelligence, traffic analysis, Big Data platform, etc. We attribute this trend as catalysed by the capability of the Cloud. We mark this trend with "(c) Data and analysis".

\section{Conclusion and Future Work}

Towards unifying the understanding of various "aaS" terms in XaaS of the Cloud, we present in this paper our survey on various forms of "aaS" extending from traditional services to the Cloud services. The current presentation focuses on listing and classifying related work by years' order to show the original routine of evolution from the past to present. We also proposed a strategy to cognitively identify the "aaS" at a series of stages of a servicelization process. We validate the categorization by applying it to identify several known facts of servicelization trends. In general, we have seen the emerging of 
"aaS" accompanying the coming of the Cloud era and evolving in branches such as Big Data, Internet of Thing, etc. We hope that this work can be inspirations for further investigation which may reveal the trend of future service migration in the Cloud and catalysis innovations in the Cloud, service brokerage [31], service choreography [65], etc. We are going to deepen the current investigation through integrating more sources such as Gartner Hype Cycles and domain knowledge ontology, etc.

\section{Acknowledgment}

The authors of the paper acknowledge the support of the National Natural Science Foundation of China (No.61363007 and 61440019), Hainan provincial Natural Science Foundation (No.20156234 and No.20156245) and the Hainan University Research program (No. KYQD1242).

\section{References}

[1] O. Agmon Ben-Yehuda, M. Ben-Yehuda, A. Schuster, and D. Tsafrir. The resource-as-a-service (raas) cloud. In Proceedings of the 4th USENIX conference on Hot Topics in Cloud Computing, pages 1212. USENIX Association, 2012.

[2] D. Agrawal, A. El Abbadi, F. Emekci, and A. Metwally. Database management as a service: Challenges and opportunities. In Data Engineering, 2009. ICDE'09. IEEE 25th International Conference on, pages 1709-1716. IEEE, 2009.

[3] T. Aho, A. Ashraf, M. Englund, J. Katajamäki, J. Koskinen, J. Lautamäki, A. Nieminen, I. Porres, and I. Turunen. Designing ide as a service. Communications of Cloud Software, 1(1), 2011.

[4] M. M. Alabbadi. Cloud computing for education and learning: Education and learning as a service (elaas). In Interactive Collaborative Learning (ICL), 2011 14th International Conference on, pages 589-594. IEEE, 2011.

[5] M. Amelung, K. Krieger, and D. Rosner. Eassessment as a service. Learning Technologies, IEEE Transactions on, 4(2):162-174, 2011.

[6] M. Armbrust, A. Fox, R. Griffith, A. D. Joseph, R. Katz, A. Konwinski, G. Lee, D. Patterson, A. Rabkin, I. Stoica, et al. A view of cloud com- puting. Communications of the ACM, 53(4):50-58, 2010.

[7] F. M. Aymerich, G. Fenu, and S. Surcis. An approach to a cloud computing network. In Applications of Digital Information and Web Technologies, 2008. ICADIWT 2008. First International Conference on the, pages 113-118. IEEE, 2008.

[8] P. Banerjee, C. Bash, R. Friedrich, P. Goldsack, B. A. Huberman, J. Manley, C. Patel, P. Ranganathan, and A. Veitch. Everything as a service: Powering the new information economy. Computer, 44(3):36-43, 2011.

[9] N. B. Beaumont, A. S. Sohal, and M. Terziovski. Comparing quality management practices in the australian service and manufacturing industries. International Journal of Quality \& Reliability Management, 14(8):814-833, 1997.

[10] D. Beimborn, T. Miletzki, and S. Wenzel. Platform as a service (paas). Business \& Information Systems Engineering, 3(6):381-384, 2011.

[11] A. Bender, N. Spring, D. Levin, and B. Bhattacharjee. Accountability as a service. SRUTI, 7:1-6, 2007.

[12] K. Bennett, P. Layzell, D. Budgen, P. Brereton, L. Macaulay, and M. Munro. Service-based software: the future for flexible software. In Software Engineering Conference, 2000. APSEC 2000. Proceedings. Seventh Asia-Pacific, pages 214-221. IEEE, 2000.

[13] T. Bitterman, P. Calyam, A. Berryman, D. E. Hudak, L. Li, A. Chalker, S. Gordon, D. Zhang, D. Cai, C. Lee, et al. Simulation as a service (smaas): a cloud-based framework to support the educational use of scientific software. International Journal of Cloud Computing 1, 3(2):177-190, 2014.

[14] A. S. Black, T. R. Sahama, and R. Gajanayake. ehealth-as-a-service (ehaas): a data-driven decision making approach in australian context. Studies in Health Technology and Informatics [e-Health-For Continuity of Care ], 205:915-919, 2014.

[15] A. Bottaro, A. Gérodolle, and P. Lalanda. Pervasive service composition in the home network. In $A d$ vanced Information Networking and Applications, 2007. AINA'07. 21st International Conference on, pages 596-603. IEEE, 2007.

[16] H. Brunelire, J. Cabot, and F. Jouault. Combining model-driven engineering and cloud computing. 2010.

[17] H. Cai, K. Zhang, M. Wang, J. Li, L. Sun, and $\mathrm{X}$. Mao. Customer centric cloud service model and 
a case study on commerce as a service. In Cloud Computing, 2009. CLOUD'09. IEEE International Conference on, pages 57-64. IEEE, 2009.

[18] A. Caminero, A. Robles-Gomez, S. Ros, L. Tobarra, R. Hernandez, R. Pastor, and M. Castro. Deconstructing remote laboratories to create laboratories as a service (laas). In Global Engineering Education Conference (EDUCON), 2014 IEEE, pages 623-629. IEEE, 2014.

[19] G. Candea, S. Bucur, and C. Zamfir. Automated software testing as a service. In Proceedings of the 1st ACM symposium on Cloud computing, pages 155-160. ACM, 2010.

[20] V. Chang. The business intelligence as a service in the cloud. Future Generation Computer Systems, 37:512-534, 2014.

[21] C.-C. Chen, L. Yuan, A. Greenberg, C.-N. Chuah, and P. Mohapatra. Routing-as-a-service (raas): A framework for tenant-directed route control in data center. In INFOCOM, 2011 Proceedings IEEE, pages 1386-1394. IEEE, 2011.

[22] J. Chen, J. Ma, N. Zhong, Y. Yao, J. Liu, R. Huang, W. Li, Z. Huang, Y. Gao, and J. Cao. Waas: wisdom as a service. Intelligent Systems, IEEE, 29(6): 40-47, 2014.

[23] Q. Chen, M. Hsu, and H. Zeller. Experience in continuous analytics as a service (caaas). In Proceedings of the 14th International Conference on Extending Database Technology, pages 509-514. ACM, 2011.

[24] B. Christophe, M. Boussard, M. Lu, A. Pastor, and V. Toubiana. The web of things vision: Things as a service and interaction patterns. Bell Labs Technical Journal, 16(1):55-61, 2011.

[25] V. Chu, R. Wong, W. Liu, F. Chen, and C. S. Perng. Traffic analysis as a service via a unified model. In Services Computing (SCC), 2014 IEEE International Conference on, pages 195-202, June 2014.

[26] T. Cicic and A. Elmokashfi. Media network as a service. Communications Magazine, IEEE, 52(8): 153-159, Aug 2014. ISSN 0163-6804.

[27] S. S. Craciunas, A. Haas, C. M. Kirsch, H. Payer, H. Röck, A. Rottmann, A. Sokolova, R. Trummer, J. Love, and R. Sengupta. Information-acquisitionas-a-service for cyber-physical cloud computing. In Proceedings of the 2nd USENIX conference on Hot topics in cloud computing, pages 14-14. USENIX Association, 2010.

[28] A. Dan, R. Johnson, and A. Arsanjani. Information as a service: Modeling and realization. In
Systems Development in SOA Environments, 2007. SDSOA'07: ICSE Workshops 2007. International Workshop on, pages 2-2. IEEE, 2007.

[29] W. Dawoud, I. Takouna, and C. Meinel. Infrastructure as a service security: Challenges and solutions. In Informatics and Systems (INFOS), 2010 The 7th International Conference on, pages 1-8. IEEE, 2010.

[30] J. Doerr, A. Benlian, J. Vetter, and T. Hess. Pricing of content services an empirical investigation of music as a service. In M. Nelson, M. Shaw, and T. Strader, editors, Sustainable e-Business Management, volume 58 of Lecture Notes in Business Information Processing, pages 13-24. Springer Berlin Heidelberg, 2010. ISBN 978-3-642-15140-8.

[31] Y. Duan, K. Huang, D. Chen, Y. Wang, A. Kattepur, and W. Du. Service value broker patterns: An empirical collection and analysis. International Journal of Networked and Distributed Computing, 2(1): 54-69, 2014.

[32] A. Dunkels, B. Gronvall, and T. Voigt. Contiki-a lightweight and flexible operating system for tiny networked sensors. In Local Computer Networks, 2004. 29th Annual IEEE International Conference on, pages 455-462. IEEE, 2004.

[33] V. Dwivedi and N. Kulkarni. Information as a service in a data analytics scenario - a case study. In Web Services, 2008. ICWS '08. IEEE International Conference on, pages 615-620, Sept 2008.

[34] S. M. Edworthy. Telemedicine in developing countries. Bmj, 323(7312):524-525, 2001.

[35] C. Emig, F. Brandt, S. Kreuzer, and S. Abeck. Identity as a service-towards a service-oriented identity management architecture. In Dependable and Adaptable Networks and Services, pages 1-8. Springer, 2007.

[36] R. Esteves. A taxonomic analysis of cloud computing. In 1st Doctoral Workshop in Complexity Sciences ISCTE-IUL/FCUL, 2011.

[37] A. Fano and A. Gershman. The future of business services in the age of ubiquitous computing. Communications of the ACM, 45(12):83-87, 2002.

[38] T. Feng, J. Bi, H. Hu, and H. Cao. Networking as a service: a cloud-based network architecture. JNW, 6(7):1084-1090, 2011.

[39] R. J. Figueiredo, P. A. Dinda, and J. A. Fortes. A case for grid computing on virtual machines. In Distributed Computing Systems, 2003. Proceedings. 23rd International Conference on, pages 550-559. IEEE, 2003. 
[40] N. Furmento, W. Lee, A. Mayer, S. Newhouse, and J. Darlington. Iceni: an open grid service architecture implemented with jini. In Proceedings of the 2002 ACM/IEEE conference on Supercomputing, pages 1-10. IEEE Computer Society Press, 2002.

[41] V. Gilart-Iglesias, F. Maciá-Pérez, A. CapellaD’Alton, and J. A. Gil-Martínez-Abarca. Industrial machines as a service: A model based on embedded devices and web services. In Industrial Informatics, 2006 IEEE International Conference on, pages 630-635. IEEE, 2006.

[42] C. Grier, L. Ballard, J. Caballero, N. Chachra, C. J. Dietrich, K. Levchenko, P. Mavrommatis, D. McCoy, A. Nappa, A. Pitsillidis, et al. Manufacturing compromise: the emergence of exploit-as-aservice. In Proceedings of the 2012 ACM conference on Computer and communications security, pages 821-832. ACM, 2012.

[43] R. L. Grossman, Y. Gu, M. Sabala, and W. Zhang. Compute and storage clouds using wide area high performance networks. Future Generation Computer Systems, 25(2):179 - 183, 2009. ISSN 0167739X.

[44] H. Hacigumus, B. Iyer, and S. Mehrotra. Providing database as a service. In Data Engineering, 2002. Proceedings. 18th International Conference on, pages 29-38. IEEE, 2002.

[45] J. Horey, E. Begoli, R. Gunasekaran, S.-H. Lim, and J. Nutaro. Big data platforms as a service: Challenges and approach. In Proceedings of the 4th USENIX Conference on Hot Topics in Cloud Ccomputing, HotCloud'12, pages 16-16, Berkeley, CA, USA, 2012. USENIX Association.

[46] B. Howe, G. Cole, E. Souroush, P. Koutris, A. Key, N. Khoussainova, and L. Battle. Database-as-aservice for long-tail science. In J. Bayard Cushing, J. French, and S. Bowers, editors, Scientific and Statistical Database Management, volume 6809 of Lecture Notes in Computer Science, pages 480489. Springer Berlin Heidelberg, 2011. ISBN 9783-642-22350-1.

[47] A. G. Howson, J. P. Kahane, P. Lauginie, and E. de Turckheim, editors. Mathematics as a Service Subject. Cambridge University Press, 1988. ISBN 9781139013505. Cambridge Books Online.

[48] W. Itani, A. Kayssi, and A. Chehab. Privacy as a service: Privacy-aware data storage and processing in cloud computing architectures. In Dependable, Autonomic and Secure Computing, 2009. DASC'09. Eighth IEEE International Conference on, pages
711-716. IEEE, 2009.

[49] B. Ives and G. P. Learmonth. The information system as a competitive weapon. Communications of the ACM, 27(12):1193-1201, 1984.

[50] S. Jansen, A. Finkelstein, and S. Brinkkemper. A sense of community: A research agenda for software ecosystems. In Software EngineeringCompanion Volume, 2009. ICSE-Companion 2009. 31st International Conference on, pages 187-190. IEEE, 2009.

[51] Y. Jin, Y. Wen, and W. Zhang. Content routing and lookup schemes using global bloom filter for content-delivery-as-a-service. Systems Journal, IEEE, 8(1):268-278, 2014.

[52] C. Jingliang, H. Keqing, M. Yutao, and Z. Neng. An approach for value as a service discovery on scientific papers big data. In Services Computing (SCC), 2014 IEEE International Conference on, pages 480-487, June 2014.

[53] N. Kailer and J. Scheff. Knowledge management as a service: cooperation between small and mediumsized enterprises (smes) and training, consulting and research institutions. Journal of European Industrial Training, 23(7):319-328, 1999.

[54] B. S. Kaliski Jr and W. Pauley. Toward risk assessment as a service in cloud environments. In Proceedings of the 2nd USENIX conference on Hot topics in cloud computing, pages 13-13. USENIX Association, 2010.

[55] L. M. Kaufman. Data security in the world of cloud computing. IEEE Security and Privacy, 7(4):61-64, jul 2009. ISSN 1540-7993.

[56] H. J. La, J. S. Her, and S. D. Kim. Framework for evaluating reusability of component-as-a-service (caas). In Principles of Engineering ServiceOriented Systems (PESOS), 2013 ICSE Workshop on, pages 41-44. IEEE, 2013.

[57] P. Laitinen, P. Ginzboorg, N. Asokan, S. Holtmanns, and V. Niemi. Extending cellular authentication as a service. In Commercialising Technology and Innovation, 2005. The First IEE International Conference on (Ref. No. 2005/11044), pages 0-90D2/4, Sept 2005.

[58] K. Lakshminarayanan, I. Stoica, S. Shenker, and J. Rexford. Routing as a service. Technical report, University of California at Berkeley, 2006.

[59] Q. Liu, G. Wang, and J. Wu. Consistency as a service: Auditing cloud consistency. Network and Service Management, IEEE Transactions on, 11(1): 25-35, 2014. 
[60] R. K. Lomotey and R. Deters. Analytics-as-aservice (aaas) tool for unstructured data mining. In Cloud Engineering (IC2E), 2014 IEEE International Conference on, pages 319-324. IEEE, 2014.

[61] Z. Maamar and Y. Badr. Social networks as a service in modern enterprises. In Current Trends in Information Technology (CTIT), 2009 International Conference on the, pages 1-5. IEEE, 2009.

[62] N. Milanovic and M. Malek. Service-oriented operating system: A key element in improving service availability. In Proceedings of the 4th International Symposium on Service Availability, ISAS '07, pages 31-42, Berlin, Heidelberg, 2007. Springer-Verlag. ISBN 978-3-540-72735-4.

[63] J. Mizusawa and N. Kitsunezaki. Hybrid network as a service: Proposal and implementation. In Ultra Modern Telecommunications and Control Systems and Workshops (ICUMT), 2011 3rd International Congress on, pages 1-5, Oct 2011.

[64] M. R. Nascimento, C. E. Rothenberg, M. R. Salvador, C. N. Corrêa, S. C. de Lucena, and M. F. Magalhães. Virtual routers as a service: the routeflow approach leveraging software-defined networks. In Proceedings of the 6th International Conference on Future Internet Technologies, pages 34-37. ACM, 2011.

[65] A. Norta, L. Ma, Y. Duan, A. Rull, M. Kõlvart, and K. Taveter. econtractual choreography-language properties towards cross-organizational business collaboration. Journal of Internet Services and Applications, 6(1):1-23, 2015.

[66] M. Ott, I. Seskar, R. Siraccusa, and M. Singh. Orbit testbed software architecture: supporting experiments as a service. In Testbeds and Research Infrastructures for the Development of Networks and Communities, 2005. Tridentcom 2005. First International Conference on, pages 136-145, Feb 2005. doi: 10.1109/TRIDNT.2005.27.

[67] M. P. Papazoglou. Service-oriented computing: Concepts, characteristics and directions. In Web Information Systems Engineering, 2003. WISE 2003. Proceedings of the Fourth International Conference on, pages 3-12. IEEE, 2003.

[68] M. P. Papazoglou, P. Traverso, I. Ricerca, and S. Tecnologica. Service-oriented computing: State of the art and research challenges. IEEE Computer, 40:2007, 2007.

[69] P. Patel, A. H. Ranabahu, and A. P. Sheth. Service level agreement in cloud computing. Technical report, Wright State University, 2009.
[70] D. Peraković, T. M. Kuljanić, and M. Musa. Xaas services as modern infrastructure of its. In 22nd International DAAAM Symposium, 2011.

[71] C. Perera, A. Zaslavsky, P. Christen, and D. Georgakopoulos. Sensing as a service model for smart cities supported by internet of things. Transactions on Emerging Telecommunications Technologies, 25 (1):81-93, 2014.

[72] R. Perrey and M. Lycett. Service-oriented architecture. In Applications and the Internet Workshops, 2003. Proceedings. 2003 Symposium on, pages 116-119. IEEE, 2003.

[73] S. Rajagopalan, B. Cully, R. O'Connor, and A. Warfield. Secondsite: disaster tolerance as a service. In ACM SIGPLAN Notices, volume 47, pages 97-108. ACM, 2012.

[74] B. P. Rimal, E. Choi, and I. Lumb. A taxonomy and survey of cloud computing systems. In INC, IMS and IDC, 2009. NCM'09. Fifth International Joint Conference on, pages 44-51. Ieee, 2009.

[75] S. Robison et al. The next wave: Everything as a service. Executive Viewpoint: www. hp. com, 2008.

[76] P. Rodríguez, D. Gallego, J. Cerviño, F. Escribano, J. Quemada, and J. Salvachúa. Vaas: Videoconference as a service. In Collaborative Computing: Networking, Applications and Worksharing, 2009. CollaborateCom 2009. 5th International Conference on, pages 1-11. IEEE, 2009.

[77] S. Sarawagi and S. H. Nagaralu. Data mining models as services on the internet. ACM SIGKDD Explorations Newsletter, 2(1):24-28, 2000.

[78] C. Senk and F. Dotzler. Biometric authentication as a service for enterprise identity management deployment: a data protection perspective. In Availability, Reliability and Security (ARES), 2011 Sixth International Conference on, pages 43-50. IEEE, 2011.

[79] A. Singh, M. Srivatsa, and L. Liu. Search-as-aservice: Outsourced search over outsourced storage. ACM Transactions on the Web (TWEB), 3(4): 13, 2009.

[80] E. Sirin, J. Hendler, and B. Parsia. Semi-automatic composition of web services using semantic descriptions. In 1st Workshop on Web Services: Modeling, Architecture and Infrastructure, pages 17-24, 2003.

[81] S. Subashini and V. Kavitha. A survey on security issues in service delivery models of cloud computing. Journal of Network and Computer Applications, 34(1):1-11, 2011. 
[82] H.-L. Truong and S. Dustdar. On analyzing and specifying concerns for data as a service. In Services Computing Conference, 2009. APSCC 2009. IEEE Asia-Pacific, pages 87-94. IEEE, 2009.

[83] H.-Y. Tsai, M. Siebenhaar, A. Miede, Y.-L. Huang, and R. Steinmetz. Threat as a service?: Virtualization's impact on cloud security. IT Professional, 14 (1):32-37, Jan 2012. ISSN 1520-9202.

[84] W.-T. Tsai, X. Sun, and J. Balasooriya. Serviceoriented cloud computing architecture. In Information Technology: New Generations (ITNG), 2010 Seventh International Conference on, pages 684689. IEEE, 2010.

[85] W. M. van der Aalst, M. Adams, A. H. ter Hofstede, M. Pesic, and H. Schonenberg. Flexibility as a service. In Database Systems for Advanced Applications, pages 319-333. Springer, 2009.

[86] A. van Deursen and W. Pieterson. The internet as a service channel in the public sector. In ICA Conference, Dresden, Germany, 2006.

[87] V. Varadharajan and U. Tupakula. Security as a service model for cloud environment. Network and Service Management, IEEE Transactions on, 11(1): 60-75, 2014.

[88] M. Viroli and A. Omicini. Coordination as a service: Ontological and formal foundation. Electronic Notes in Theoretical Computer Science, 68 (3):457-482, 2003.

[89] M. Wang, K. Bandara, and C. Pahl. Process as a service distributed multi-tenant policy-based process runtime governance. In Services Computing (SCC), 2010 IEEE International Conference on, pages 578-585, July 2010.

[90] R. Wang, S. Chen, X. Wang, and S. Qadeer. How to shop for free online-security analysis of cashieras-a-service based web stores. In Security and Privacy (SP), 2011 IEEE Symposium on, pages 465480. IEEE, 2011.
[91] C. Weinhardt, B. Blau, D. I. N. Borissov, D. M. T. Meinl, D. I. W. W. Michalk, and J. St0208er. Cloud computing a classification, business models, and research directions. Business \& Information Systems Engineering, 1(5):391-399, 2009.

[92] P. Wong, Z. He, and E. Lo. Parallel analytics as a service. In Proceedings of the 2013 international conference on Management of data, pages 25-36. ACM, 2013.

[93] T. Wood, E. Cecchet, K. Ramakrishnan, P. Shenoy, J. Van Der Merwe, and A. Venkataramani. Disaster recovery as a cloud service: Economic benefits \& deployment challenges. In Proceedings of the $2 n d$ USENIX conference on Hot topics in cloud computing, pages 8-8. USENIX Association, 2010.

[94] J. Wu, L. Ping, X. Ge, Y. Wang, and J. Fu. Cloud storage as the infrastructure of cloud computing. In Intelligent Computing and Cognitive Informatics (ICICCI), 2010 International Conference on, pages 380-383. IEEE, 2010.

[95] S. Xu and W. Zhang. Knowledge as a service and knowledge breaching. In Services Computing, 2005 IEEE International Conference on, volume 1, pages 87-94. IEEE, 2005.

[96] Q. Yao, X. Han, X. K. Ma, Y. F. Xue, Y. J. Chen, and J. S. Li. Cloud-based hospital information system as a service for grassroots healthcare institutions. Journal of Medical Systems, 38(9):797-811, 2014.

[97] L. Yu, W.-T. Tsai, X. Chen, L. Liu, Y. Zhao, L. Tang, and W. Zhao. Testing as a service over cloud. In Service Oriented System Engineering (SOSE), 2010 Fifth IEEE International Symposium on, pages 181-188. Ieee, 2010.

[98] S. A. Zargari and A. Smith. Policing as a service in the cloud. In Emerging Intelligent Data and Web Technologies (EIDWT), 2013 Fourth International Conference on, pages 589-596. IEEE, 2013. 